\title{
Chronology of the Late Period
}

Post-Imperial Epoch

Twenty-first Dynasty

Twenty-second Dynasty

Sheshonq I

Osorkon I

Osorkon II

Osorkon IV

Twenty-third Dynasty

Iuput II

Twenty-fourth Dynasty

Tefnakht

Twenty-fifth (Nubian) Dynasty in Egypt

Piye

Shabako

Taharqa

Tantamani

Saite Period: Twenty-sixth Dynasty

Psamtik I

Necho II

Psamtik II

Apries

Amasis

Psamtik III

First Persian Domination: Twenty-seventh Dynasty

Cambyses

Darius I

Xerxes

Artaxerxes

Darius II

Last Independence: Dynasties 28-30

Twenty-eighth Dynasty

Twenty-ninth Dynasty са. $1070-656$ в.с.

1070-945

945-715

945-913

916-904

$890-860$

$735-712$

818-715

745-725

$740-715$

$740-718$

$753-65^{6}$

753-713

$713-698$

$698-664$

$664-656$

664-525

664-610

610-595

595-589

589-570

570-526

526-525

525-404 525-522

52 1-486 486-466

$4^{6} 5-4^{24}$

424-4O4

404-341

404-398

$398-378$ 
Thirtieth Dynasty

Nectanebo I (Nekht-nebef)

378-341

Nectanebo II (Nekht-hor-heb)

Second Persian Domination

$34^{1-332}$

Artaxerxes III (Ochos)

$34^{1-33^{8}}$

Darius III (Codoman)

Alexander the Great

$33^{2-323}$

Ptolemaic Period

323-30

Ptolemy I Soter 323-282

Ptolemy V Epiphanes 205-180

Ptolemy XII Neos Dionysos

8o- 51

Cleopatra VII Philopator

$5^{1-30}$

Roman Period

3O B.C. -324 A.D.

Note: Only kings mentioned in the texts and comments of this volume are listed here. Note also that some of the dates are conjectural. 